American Journal of Applied Sciences 2 (4): 877-880, 2005

ISSN 1546-9239

(C) Science Publications, 2005

\title{
Residual Defects in Silicon Implanted with Boron and Phosphorous Ions
}

\author{
M. Jadan \\ Department of Basic Sciences, Tafila Applied University College \\ Al-Balqa' Applied University, P.O. Box 40, AL-Eys_66141, Tafila, Jordan
}

\begin{abstract}
Accumulation of radiation defects in $\mathrm{Si}$ implanted with $\mathrm{B}^{+}$or $\mathrm{P}^{+}$ions, and formation of the residual extended defects (dislocation loops, rod-like defects) in the process of successive thermal treatment have been studied. The anomalies observed in the formation of the extended defects are associated with the elastic stresses in the damaged regions affecting the process of radiation defects clustering.
\end{abstract}

Key words: Accumulation of radiation defects, residual, dislocation loops

\section{INTRODUCTION}

Ion implantation in silicon brings about a great number of radiation defects stable at room temperatures. To eliminate the defects and to introduce the implanted impurity into the lattice nodes, hightemperature annealing of the implanted structures is carried out. However, upon annealing a great part of the radiation defects is converted to the extended ones such as dislocation loops and rod-like defects $\{113\}$. These defects are characterized by deep levels within the band gap of silicon, leading to degradation of the performance of microelectronic devices and affecting the yield.

The formation of residual defects is threshold in nature, i.e. upon thermal treatment the extended defects are formed at a particular critical concentration of the point defects resulting from implantation. Quite surprising, it has been found that extended defects are formed in silicon implanted with light $\mathrm{B}^{+}$ions, whose threshold dose for the extended defect formation turned to be the same $\left(\sim 10^{14} \mathrm{~cm}^{-2}\right)$ as for the average-weight ions $\left(\mathrm{Si}^{+}, \mathrm{P}^{+}\right)^{[1]}$, despite significant differences in the insertion efficiency of stable radiation defects. In an effort to explain this coincidence, in some works ${ }^{[2,3]}$ it has been suggested that during annealing boron atoms are effective segregation centers of point defects. And in other works ${ }^{[1]}$ this phenomenon has been attributed to low efficiency of the extended defect formation on irradiation with $\mathrm{Si}^{+}$and $\mathrm{P}^{+}$ions due to the amorphous structure of the nucleus in the damaged region rather than to the high-efficiency formation of extended defects with the use of $\mathrm{B}^{+}$ions. Upon thermal treatment, amorphous inclusions are crystallized without emission of the point defects needed for the formation of extended ones. At the present time, however, it is well known that initially in silicon the Defect Cluster Regions (DCR) created by implantation of the average mass ions are not amorphous.

This paper is a study accumulation of the radiation defects in silicon on implantation with $\mathrm{P}^{+}$and $\mathrm{B}^{+}$ions.
The processes of residual defect formation upon annealing are also studied with the use of Transmission Electron Microscopy (TEM) methods.

\section{MATERIALS AND METHODS}

The samples of $p$-type $\mathrm{Si}$ with initial resistivity of $10 \Omega \mathrm{cm}$, were implanted with $\mathrm{P}^{+}$and $\mathrm{B}^{+}$ ions with an energy of 200 and $100 \mathrm{keV}$, respectively. The scanning beam implantation was realized with the effective ion current density amounting to $0.2 \mu \mathrm{A} \mathrm{cm}^{-2}$. The accumulation of radiation defects was determined from the change of the lattice constant $\Delta \mathrm{a}$ in the implanted layer. The $\Delta \mathrm{a}$ values were measured by the angle difference between diffraction maxima of the $\mathrm{X}$ ray reflection from the implanted layer and the substrate. The diffraction curves were measured by a double crystal X-ray spectrometer for a parallel position of the crystal-monochromator with the sample using $\mathrm{CuK} \alpha_{1}$ irradiation in the fourth order of at (111) reflection. The accuracy of the determination of the $\Delta \mathrm{a}$ was $1 \times 10^{-6} \mathrm{~nm}$. The concentration of the radiation defects was estimated from the $\Delta \mathrm{a}$ values taking into account the value of the atom displacement in the regions of prevalent defects which is equal to 0.02 $\mathrm{nm}^{[4]}$.

Radiation defects in the implanted silicon have been studied using the Electron Paramagnetic Resonance (EPR) method. Spectra were recorded with the help of Varian spectrometer over a $3 \mathrm{~cm}$ wavelength region of MW radiation. Annealing of the implanted silicon structures was performed in sealed evacuated quartz tubes at $900^{\circ} \mathrm{C}$. Extended defects have been investigated by the EM-100 electron microscope with the use of TEM method.

\section{RESULTS AND DISCUSSION}

Figure 1a shows the changes in lattice constant $\Delta \mathrm{a}$ of a silicon layer implanted with $\mathrm{P}^{+}$ions as a function of the dose. The observed increase in the lattice 
constant is due to the introduction of point radiation defects known to be accumulated mainly in DCR. For doses above $1.10 \mathrm{~cm}^{-2}$ one can observe deviation from the linear dependence of $\Delta \mathrm{a}(\Phi)$ attributed to the enhanced annihilation of the defects for the overlapping displacement cascades formed with the previously introduced DCR. Then a bend in the curve $\triangle \mathrm{a}(\Phi)$ is observed. This bend with a simultaneous reduction in the X-ray reflection intensity from the implanted layer is associated with the beginning amorphization, as evidenced by the EPR spectra exhibiting an isotropic line with g-factor of 2.0055 .

The formation of displacement cascades in silicon implanted with the average mass ions has probabilistic character. When $\mathrm{N}$ ions are introduced into the crystal unit volume $\mathrm{V}$, the probability $\mathrm{P}$ of falling $\mathrm{n}$ ions within the chosen volume $v$ is determined by Poisson probabilities:

$P(N, v, n)=\exp (-N v) \frac{(N v)^{n}}{n !}$

where, $v \approx v_{\mathrm{k}}$ is the averaged volume of the defect cluster region. Succession of $n$ ions entering volume $v$ results the $n$-fold overlapping damaged regions. Since the probability of cascade formation at any point within the volume $\mathrm{V}$ is independent of the irradiation dose, the volume occupied by the n-fold overlapping damaged regions are determined by the Poisson probability. As the formation efficiency of displacement cascade and hence DCR per one incident ion is not necessarily equal to unity, we obtain $\mathrm{N}=\mathrm{K} \Phi$, where, $\Phi$ is the ion irradiation dose and $\mathrm{K}$ - number of the displacement cascades formed for a single ion. Fig. $1 \mathrm{~b}$ presents the probability functions $\mathrm{P}(\mathrm{Nv})$ for $\mathrm{n}=1,2,3,4$.

Numerical values of $\mathrm{P}(\mathrm{Nv})$ determine a relative volume occupied by the isolated $(n=1)$ and $n$-fold overlapping $(\mathrm{n}=2,3,4 \ldots)$ DCR. Assuming that deviation from the linear dependence $\Delta \mathrm{a}(\Phi)$ is due to the twofold overlapping of the clusters, abscissae coordinates of $\mathrm{P}(\mathrm{Nv})$ and $\Delta \mathrm{a}(\Phi)$ may be brought into coincidence. For low values of Nv Poisson probability is equal to (Nv) $/ n$ !, i.e. At the initial stage of irradiation the number of the introduced nonoverlapping $(n=1)$ volumes $v$ of DCR increases linearly with the ion dose. With the onset of overlapping, for volumes $v$ at $n=1$ the function $\mathrm{P}(\mathrm{Nv})$ is deviated from linearity (Fig. 1b, curve 1) following the experimental function $\Delta \mathrm{a}(\Phi)$ (Fig. 1a). This enables one to determine for each irradiation dose the defect concentration in the implanted layer, relative volumes occupied both by the non-overlapping and n-fold overlapping DCR as well as the concentration of the defects in DCR.

Similar estimates have been obtained for the case of silicon irradiated with $\mathrm{B}^{+}$ions too. The results are summarized in the Table 1.
Table 1: Threshold Dose for the Formation of Residual Defects $D_{\text {th }}$, Defect Concentration in the Layer Implanted with this Dose $\mathrm{C}_{\mathrm{cr}}$, Relative Volume Occupied by DCR Non-overlapping at the Threshold Dose $\mathrm{V}^{\mathrm{DCR}}$ and Defect Concentration in DCR $\mathrm{C}^{\mathrm{DCR}}$ on Irradiation of $\mathrm{Si}$ by $\mathrm{P}^{+}$and $\mathrm{B}^{+}$Ions

\begin{tabular}{lllll}
\hline Ion & Dth $\left(\mathrm{cm}^{-2}\right)$ & $\mathrm{C}_{\mathrm{cr}}\left(\mathrm{cm}^{-2}\right)$ & $\mathrm{V}^{\mathrm{DCR}}(\%)$ & $\mathrm{C}^{\mathrm{DCR}},\left(\mathrm{cm}^{-3}\right)$ \\
\hline $\mathrm{B}^{+}$ & $9 \cdot 10^{13}$ & $4 \cdot 10^{18}$ & 4 & $1 \cdot 10^{20}$ \\
$\mathrm{P}^{+}$ & $8 \cdot 10^{13}$ & $4 \cdot 10^{19}$ & 10 & $4 \cdot 10^{20}$ \\
\hline
\end{tabular}

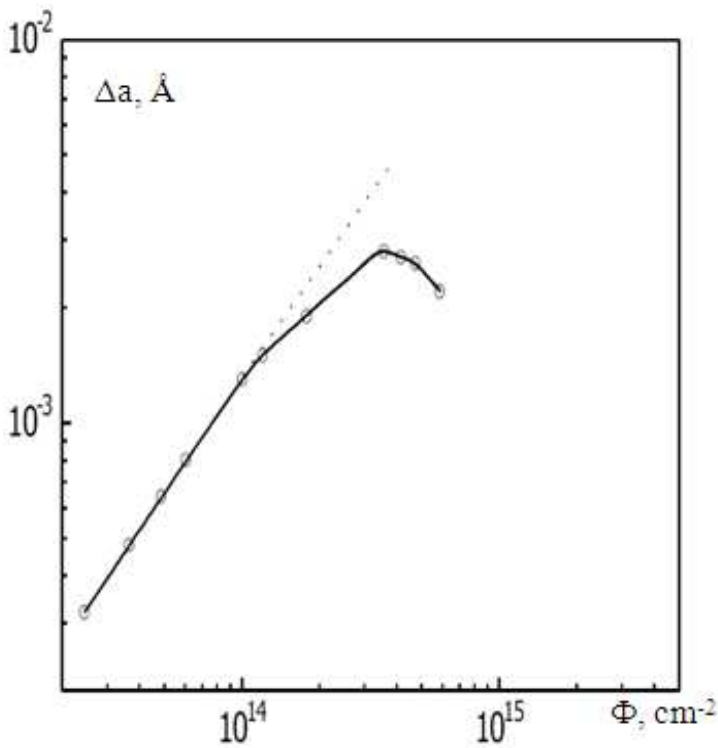

(a)

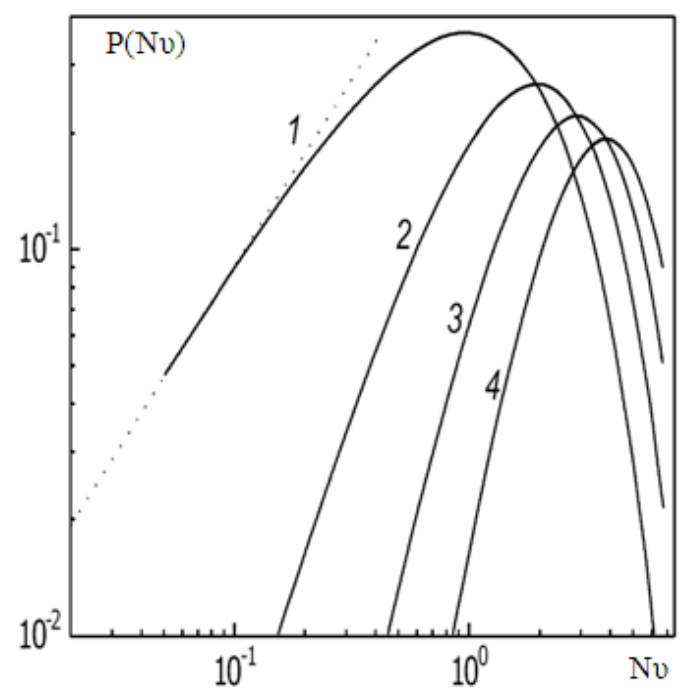

(b)

Fig. 1: (a) Lattice Constant in Silicon Implanted with $\mathrm{P}^{+}$ Ions as a Function of the Dose b) Distributions of Relative Volumes Occupied by the n-fold Overlapping DCR

Electron microscopy has demonstrated that at the implantation doses of $\mathrm{P}^{+}$and $\mathrm{B}^{+}$ions up to $1.10^{14} \mathrm{~cm}^{-2}$. The residual defects in silicon are not revealed, in accordance with the data given in ${ }^{[1]}$. Small and actually 
identical concentrations of the residual defects have been observed after implantation with a dose of $1.5 \cdot 10^{14}$ $\mathrm{cm}^{-2}$. At high doses $\left(5 \cdot 10^{14} \mathrm{~cm}^{-2}\right)$, the concentrations of the extended defects formed on irradiation by different ions differ appreciably. In case of heavier $\mathrm{P}^{+}$ions (Fig. 2a) more radiation defects are created, and the concentration of the residual defects is two orders as high as that for $\mathrm{B}^{+}$ions (Fig. 2b). At the same time, the threshold doses associated with the formation of extended defects in both cases are identical.

At the ion irradiation doses close to the threshold for the formation of extended defects $\left(\sim 1 \cdot 10^{14} \mathrm{~cm}^{-2}\right)$ the concentration of radiation defects in the implanted silicon layer irradiated by $\mathrm{P}^{+}$ions is 10 times as high as in the layer irradiated by $\mathrm{B}^{+}$ions (Table 1). It is important that on irradiation by $\mathrm{B}^{+}$ions the residual defects occur in the implanted layer when DCR occupy $4 \%$ of its volume. And in case of $\mathrm{P}^{+}, 10 \%$ of the implanted layer should be occupied by DCR. The average concentration of radiation defects in DCR of silicon upon boron implantation comes to $1 \cdot 10^{20} \mathrm{~cm}^{-3}$, whereas on implantation with phosphorous - to $4 \cdot 10^{20}$ $\mathrm{cm}^{-3}$. In this way extended defects in $\mathrm{Si}$ are formed more readily with the formation of DCR characterized by a low local density of the defects $\left(\mathrm{B}^{+}\right.$irradiation) as compared to the high-density in DCR ( $\mathrm{P}^{+}$irradiation).

It is well known that radiation defects lead to an increased lattice constant of Si. Because of this, in DCR with high local concentration of the defects the lattice constant should be greater than the average value in the implanted layer. As DCR is surrounded by a more perfect matrix, elastic stresses at the interface seem to be an additional driving force for the point defect migration from the defect cluster region during annealing. The processes of defect removal from the clustering regions and residual defect formation are concurrent. When the number of DCR is small, emission of the defects is predominant, no residual defects are formed. With increased number of DCR in the implanted layer the associated elastic-stress fields are overlapping, being partially compensated. In case of silicon implantation with phosphorous ions excess of the radiation defect concentration in DCR over the average for the whole implanted layer is much greater than in case of boron. Because of this, DCR is encircled by higher elastic stresses, the partial compensation of which necessitates higher density of DCR. When implanted with lighter ions, silicon exhibits lower concentration of the defects in DCR. Besides, quite a number of defects are formed beyond DCR. In consequence the compensation of elastic stresses may be realized for lesser density of DCR. The idea concerning the effect exerted by elastic stresses on the residual defect formation is supported by the results presented by Coffa et al. ${ }^{[5]}$. In this work anisotropy in the distribution of residual defects has been attributed to elastic stresses at the boundaries of the implanted.

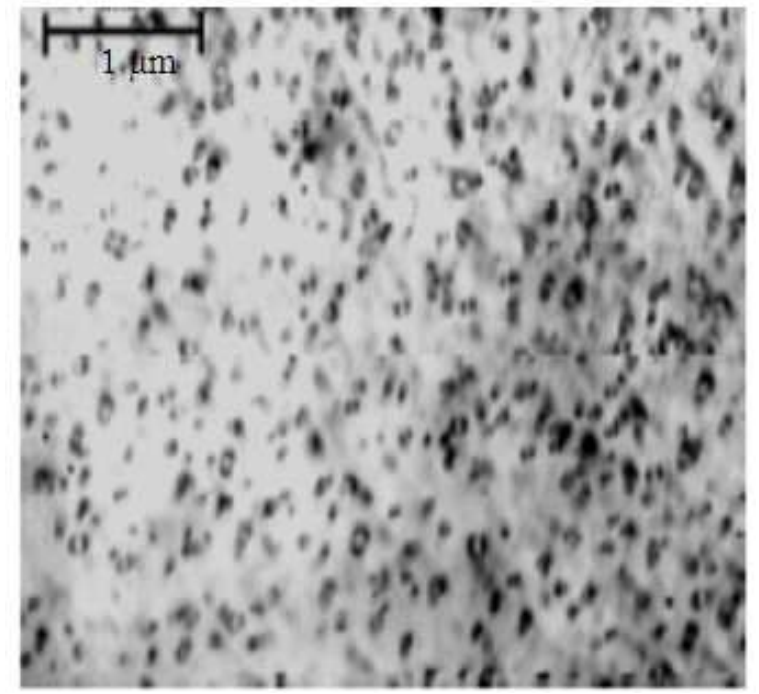

(a)

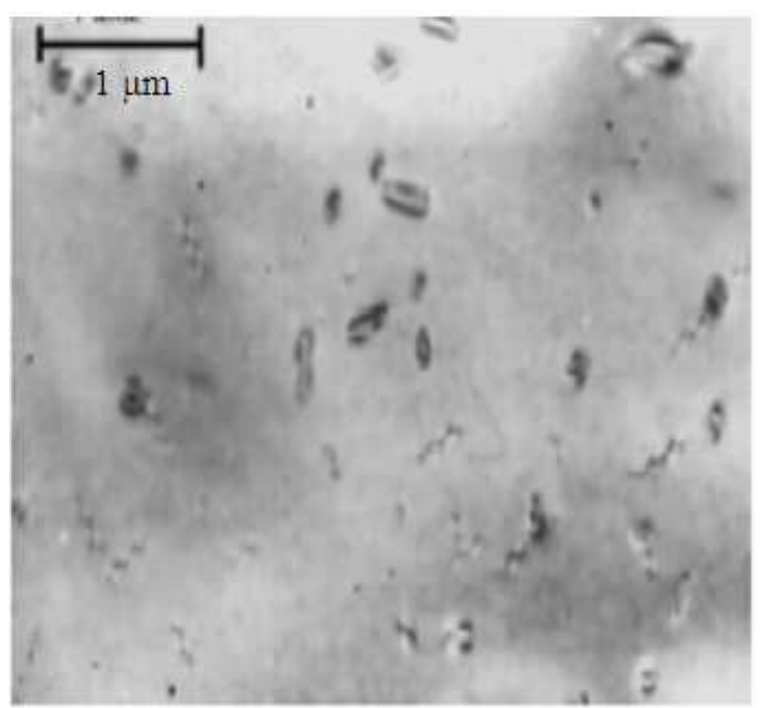

(b)

Fig. 2: $\quad$ TEM Images of Si Implanted with $\mathrm{P}^{+}$(a) and $\mathrm{B}^{+}$(b) Ions and Annealed at $900^{\circ} \mathrm{C}$ during a Period of $20 \mathrm{~min}$. Ion Irradiation Dose: $5 \cdot 10^{14} \mathrm{~cm}^{-2}$

Islands created by ion irradiation through the windows in $\mathrm{SiO}_{2}$ or $\mathrm{Si}_{3} \mathrm{~N}_{4}$ masks. A theoretical study into the effect of elastic stresses on the stability of dislocation loops is given by Legotin et al. ${ }^{[6]}$. It has been shown that the stability of a loop having the critical radius is dependent on a sign of the elastic stresses.

It is well known that an increase in the ion energy leads to the reduced cross section of elastic interactions, and the defects are introduced into the implanted layer more homogeneously. This should be shown up by the threshold for the residual defect formation. Actually, when silicon is irradiated by the intrinsic ions with an energy of $200 \mathrm{keV}$, a threshold dose comes to $8 \cdot 10^{13}$ 
$\mathrm{cm}^{-2}$, and with an energy of $1.2 \mathrm{MeV}$ - to $1 \cdot 10^{13} \mathrm{~cm}^{-2[7]}$, though the volume density of radiation defects in this case even lowers.

\section{CONCLUSION}

Anomalies in the formation of residual defects at thermal treatment of silicon implanted with light-weight $\mathrm{B}^{+}$and heavier $\mathrm{P}^{+}$ions, i.e. Identical threshold doses of the residual defect formation with the appreciable (10fold) differences in the efficiencies of introducing the stable radiation defects, may be attributed to the elastic stresses, created by the Defect Cluster Regions (DCR) which affect clustering of point defects.

\section{REFERENCES}

1. Schentelkamp, R.J., J.S. Custer, J.R. Liefting, W.X. Lu and F.W. Saris, 1991. Pre-amorphization damage in ion-implanted silicon. Mater. Sci. Rep., 6(7-8): 275-366.

2. Sechan, K. and J. Washburn, 1972. Formation of extended defects in boron implanted silicon. Rad. Effects, 14 (2): 267-269.
3. Sechan, K. and J. Washburn, 1975. Effect of ion species on residual defect formation. Rad. Effects, 26 (1): 31-33.

4. Zhevno, A.N., V.V. Sidorik and V.D. Tkachev, 1976. Atomic displacements and redistribution of electron density in the region of paramagnetic defects in silicon. Proc. Ac. Sc. Belarus, 20 (5): 409-411.

5. Coffa, S., J. La Magna, V. Privitera and G. Mannino, 1988. Surface stress and impurity effects on room temperature migration of ion-beamgenerated point defects. Appl. Phys. Lett., 73(11): 1571-1573.

6. Legotin, D.L., O.V. Bubnovskaya and N.A. Tyapunina, 1996. Simulation of dislocation loop behavior in the inhomogeneous fields. J. Moscow Univ., 3(1): 58-64.

7. Coffa, S., S. Libertine and C. Spinella, 2000. Effect of ion energy on extended defect formation. Appl. Phys. Lett., 76(2): 321-323. 